\title{
Serial Thick Section Gas Cluster Ion Beam Scanning Electron Microscopy
}

\author{
Kenneth J. Hayworth ${ }^{1}$, David Peale ${ }^{1}$, Zhiyuan $\mathrm{Lu}^{2}$, C. Shan $\mathrm{Xu}^{1}$ and Harald F.Hess ${ }^{1}$ \\ 1. Janelia Research Campus, Howard Hughes Medical Institute, Ashburn, United States. \\ 2. Department of Psychology and Neuroscience, Dalhousie University, Halifax, Canada.
}

Focused Ion Beam Scanning Electron Microscopy (FIB-SEM) is used to volume image heavy metalstained, plastic-embedded biological samples with resolutions below $10 \times 10 \times 10 \mathrm{~nm}$, an ability that is especially important in connectomics [1]. FIB-SEM samples are typically restricted to be $<50 \mu \mathrm{m}$ in the direction of the FIB beam because glancing angle milling results in artifacts over longer distances [1]. Removal rate is also restricted due to a current/spot size tradeoff. These limitations are especially problematic when one contemplates combining FIB with the increased speed offered by multibeam SEMs like the 91 beam Zeiss MultiSEM [2]. The MultiSEM's minimum field of view is $\sim 180 \mu \mathrm{m}$, and its imaging rate is approximately two orders of magnitude faster than FIB's milling rate. These considerations appear to preclude the integration of traditional FIB milling with MultiSEM imaging.

To overcome these limitations we chose to develop a broad ion beam milling approach using Gas Cluster Ion Beams (GCIB). GCIB delivers low-energy atoms to a surface and therefor does not require the use of a glancing angle. GCIB has been used for semiconductor polishing and for profiling in mass spectroscopy [3]. We attached a GCIB-10s gun from Ionoptika to a Zeiss Ultra SEM. Using a 10kV beam of Ar2000 (clusters of 2000 argon atoms), we verified that smooth, sub-10nm removal was possible from the surface of $100 \mathrm{~nm}$ thick tissue sections. In order to obtain surfaces sufficiently smooth to produce quality secondary electron (SE) images (using $1.2 \mathrm{kV}$ landing energy and InLens detection meant to mimic MultiSEM conditions) we found it was beneficial to angle the sample so that the GCIB beam made a $\sim 30^{\circ}$ angle to its surface, and to rotate the sample during milling. Using this technique we were able to volume image 100nm thick sections of tissue embedded in Durcupan and Spurr's resins but noticed that Epon samples produced surfaces too rough for SE imaging.

Sections thicker than 100nm were insufficiently conductive for quality SE imaging. To overcome this we 'precooked' thicker sections with a high energy electron beam to increase their bulk conductivity [4]. We have verified that sections as thick as $10 \mu \mathrm{m}$ can be GCIB-SEM volume imaged using SE as long as they are precooked with a $30 \mathrm{kV}$ electron beam.

For connectomics, GCIB-SEM could be used as follows: A tissue sample would first be cut into thick sections (e.g. $1 \mu \mathrm{m}$ thick using traditional microtomy, $>20 \mu \mathrm{m}$ using a diamond hot knife [5]) which would be laid out on the surface of a silicon wafer and precooked with a high voltage electron flood gun to increase bulk conductivity. This wafer would then be cycled between SEM imaging and GCIB milling acquiring one image of each section's milled surface for every $\sim 10 \mathrm{~nm}$ of surface removal.

As a demonstration we collected three sequential $1 \mu \mathrm{m}$ thick sections of Drosophila brain tissue on silicon. We precooked regions of each section $\left(10,000 \mu \mathrm{m}^{2}\right.$ area, $10 \mathrm{nA}, 4 \mathrm{hrs}$ at $10 \mathrm{kV}, 2 \mathrm{hrs}$ at $\left.6 \mathrm{kV}\right)$ to enhance their conductivity. Then we performed 250 mill/image cycles in order to completely image through all three sections (GCIB: 20nA of Ar2000 at $10 \mathrm{kV}$ spread over a $10 \mathrm{~mm}^{2}$ area, $26^{\circ}$ angle between beam and plane of surface, $360^{\circ}$ rotation, $900 \mathrm{~s}$ ( $4 \mathrm{~nm}$ removal) per cycle; SEM: $1.2 \mathrm{kV}, 2 \mathrm{nA}$ electron beam, InLens SE detection, $6 \mathrm{~nm}$ pixels, $2 \mathrm{MHz}$ ). Figure 1A shows one SEM image of this 
dataset which was acquired after many previous rounds of GCIB milling. Neuronal processes and synaptic features are clearly visible. Figure 1B shows a cross section through each of the three $1 \mu \mathrm{m}$ thick sections. The unevenness of the bottoms of each section is a result of GCIB milling rate variance $(\sim 10 \%)$ between different regions of the same section. This unevenness was computationally flattened prior to stitching the three $1 \mu \mathrm{m}$ thick volumes together into a single volume suitable for connectomic tracing as shown in Figure 2. Since high current GCIB sources are available that can easily keep up with MultiSEM imaging rates we believe that this technique of Serial Thick Section GCIB-SEM may offer a promising approach to large scale connectomics.

\section{References:}

[1] CS Xu et al, Elife 6 (2017), p. e25916.

[2] T Keman, T Garbowski and D Zeidler, Proc. of SPIE 9658 (2015), p. 965807-1.

[3] I Yamada et al, Mater. Sci. Eng. R Rep 34 (2001), p. 231.

[4] L Calcagno, G Compagnini and G Foti, Nucl. Instr. Meth. Phys. Res. B 65 (1992), p. 413.

[5] KJ Hayworth et al, Nat. Methods 12 (2015), p. 319.
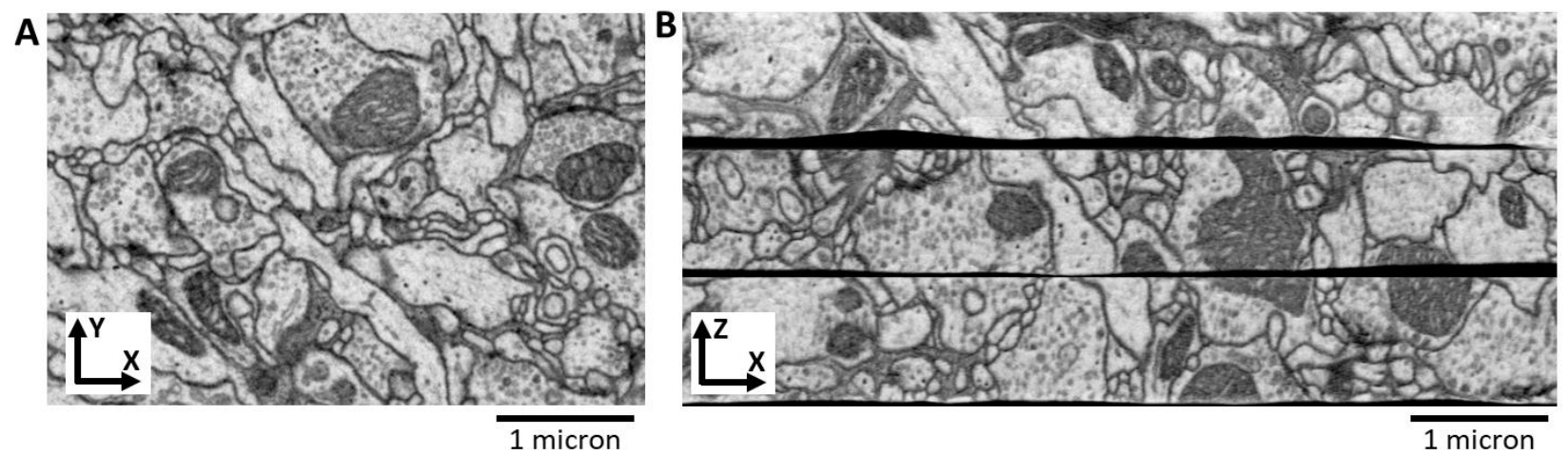

Figure 1. GCIB-SEM imaging. (A) SE image after multiple rounds of GCIB milling. (B) Cross section through dataset of three consecutive $1 \mu \mathrm{m}$ thick sections prior to computational flattening.

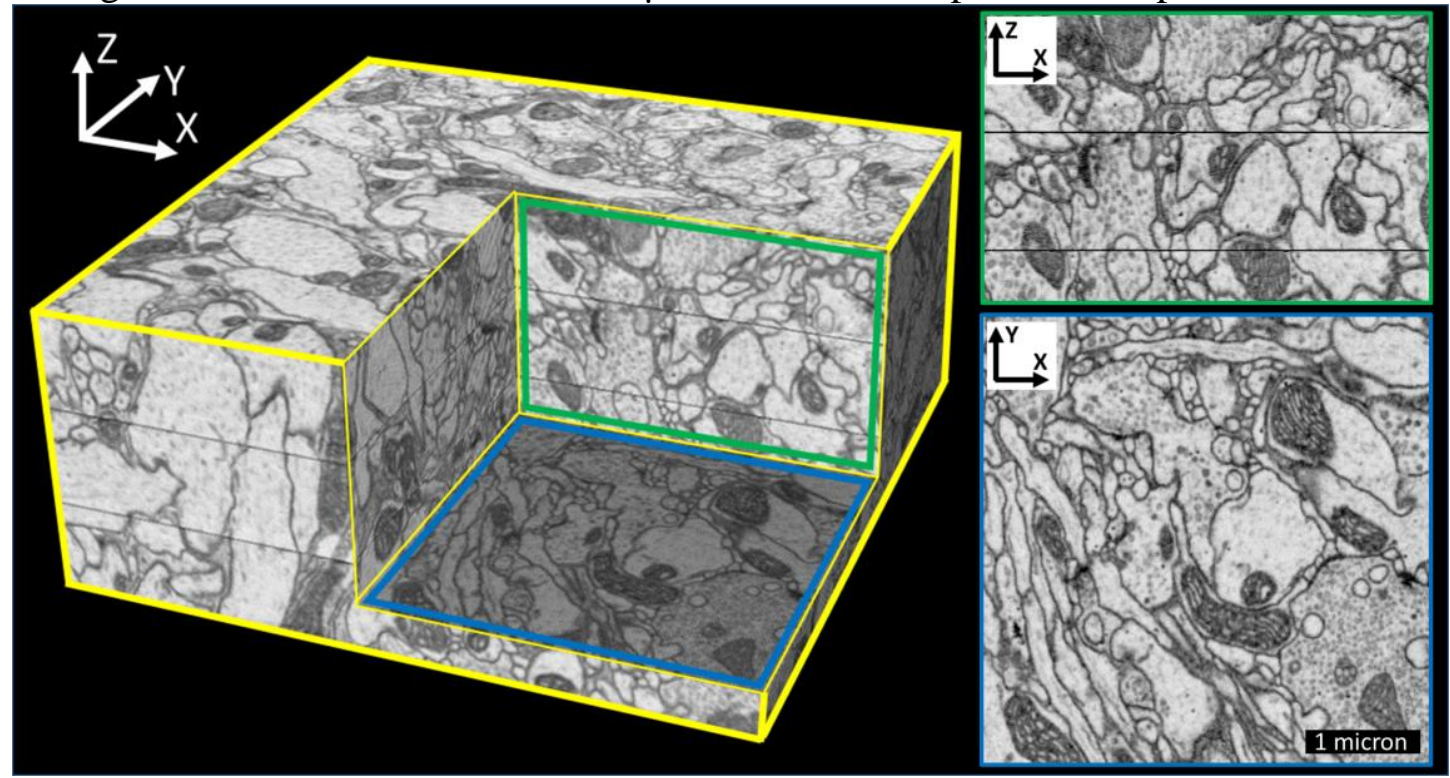

Figure 2. Final GCIB-SEM dataset after computationally flattening and volume-stitching the three consecutive $1 \mu \mathrm{m}$ thick sections together. 